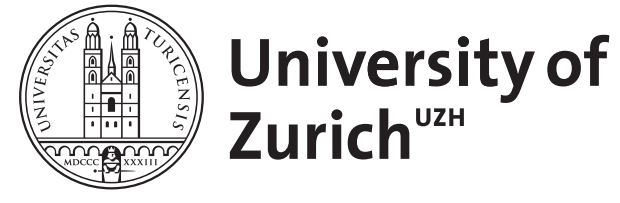
Archive

University of Zurich

University Library

Strickhofstrasse 39

CH-8057 Zurich

www.zora.uzh.ch

Year: 2006

\title{
Antiphon: Zur Resonanz des Lärms in der Geschichte
}

Dommann, Monika

DOI: https://doi.org/10.7788/ha.2006.14.1.133

Posted at the Zurich Open Repository and Archive, University of Zurich

ZORA URL: https://doi.org/10.5167/uzh-77640

Journal Article

Published Version

Originally published at:

Dommann, Monika (2006). Antiphon: Zur Resonanz des Lärms in der Geschichte. Historische Anthropologie, 14(1):133-146.

DOI: https://doi.org/10.7788/ha.2006.14.1.133 


\title{
F o r u m
}

\section{Antiphon: Zur Resonanz des Lärms in der Geschichte ${ }^{1}$}

\author{
von Monika Dommann
}

Im Jahre 1885 wird im Reichspatentamt in Berlin ein kleines Gerät angemeldet das „Antiphon“. In einer Broschüre erklärt ihr Erfinder die Funktion der, mit einem metallenen Stiel versehenen Kugeln aus Hartgummi, die zwecks „Unhörbarmachens von Tönen und Geräuschen" in den äußeren Gehörgang gesteckt werden. ${ }^{2}$ Mittels eines Karabinerhakens an einer Uhrkette befestigt, sind sie im modernen mobilen Leben flexibel anwendbar. Der „Rettungsapparat gegen den Hörzwang" gehört $\mathrm{zu}$ jenen Erfindungen, die bald von der technischen Entwicklung überholt werden: Der Drogist Maximilian Negwer ersetzt 1907 das harte Material, das einen unangenehmen Druck auf die Ohrmuschel ausübt, durch in Vaseline und Paraffinwachs getränkte Watte und lanciert mit dem „Ohropax“3 eines jener vielen kleinen ,Werkzeuge" der Kultur ${ }^{4}$, die gegen das sinnliche Exponiert-Sein entwickelt worden waren. Das „Antiphon“ setzt an bei einer der primären Eigenschaften des akustischen Sinnes: Es fehlt ihm jene natürliche Vorrich- tung, um sich gegen außen abzuschließen, die das Augenlid für den Sehsinn darstellt. Das „Antiphon“ wäre wohl endgültig vergessen, wenn nicht seine Begleitbroschüre im kulturellen Gedächtnis gespeichert worden wäre: In der alphabetischen Logik der Karteikarten in Bibliotheken erscheint der Text ganz zu Beginn einer Reihe von Texten, die unter dem Stichwort Lärm eingereiht sind. Die Broschüre ist mehr denn eine bloße Produktbeschreibung, dafür ist sie mit rund 50 Seiten viel zu umfangreich. Sie ist ein Pamphlet gegen Lärm, und gleichzeitig gegen die Stadt, die Masse, die Ungebildeten, die Unmündigen und die Unnützen, kurz: gegen jene, die ihn verursachten und selbst unempfindlich seien. Den Gebildeten, Gesitteten, Erwachsenen und Nützlichen andererseits drohten die höchsten aller bürgerlicher Güter, Freiheit und Eigentum, entrissen zu werden. Lärm das sind die „kreischenden Strassenverkäufer“, der „peitschenknallende Knecht“", die „trommelnden Kinder“ und die „musizierenden Almosensammler". 5 Keine Angst

1 Der Essay geht auf einen Vortrag zurück, den ich am 19. März 2004 an der vom Schweizerischen Werkbund Ortsgruppe Zürich veranstalteten Tagung „Lärm ist das Geräusch der andern" gehalten habe. Ich danke Michael Guggenheim und Jakob Tanner für unentbehrliche Hinweise und Kritik.

2 M. Plessner, Das Antiphon. Ein Apparat zum Unhörbarmachen von Tönen und Geräuschen, Ratenow 1885.

3 Vgl. http://www.ohropax.de/ (19.12.2005).

4 Sigmund Freud, Das Unbehagen in der Kultur, in: Alexander Mitscherlich/Angela Richards/ James Strachey (Hg.), Sigmund Freud: Studienausgabe, Band IX. Fragen der Gesellschaft, Ursprünge der Religion, [Wien 1930] Frankfurt a. M. 1979, 221.

5 Plessner, Das Antiphon, 12. 
des Bürgertums wird ausgelassen: Weder der Alkohol, noch die Gefahr eines evolutionären Rückfalls des „Pöbels“ bzw. dessen physiognomische Ähnlichkeit mit den Brüllaffen. ${ }^{6}$

Der Begleittext zum Antiphon ist nicht originell. Pamphlete gegen Lärm hat man schon früher anderswo lesen können. Der Text stellt sich denn auch in eine illustre Reihe mit Arthur Schopenhauer, Charles Babbage und Thomas Carlyle. Für Schopenhauer ist Lärm in seinem 1851 veröffentlichten Text „Über Lerm und Geräusch“ „das wahnhaft infernale Peitschenknallen" in den Städten. Lärm ist eine Waffe der Handarbeiter im Krieg mit den Kopfarbeitern. ${ }^{7}$ Jeder einzelne Knall greife die Konzentrationsfähigkeit des Geistes an und unterbreche Gedanken. Im von Charles Babbage, dem Mathematiker und Erfinder von mechanischen Rechenmaschinen, 1864 verfassten Pamphlet ,Street Nuisances" ist Lärm Straßenmusik. ${ }^{8}$ Hinter dem Phänomen Straßenmusik entlarvt er ein komplexes System im Umkreis der Unterschichten: Public-houses, Gin-shops, Bedienstete, Tavernenbesitzer, Kinder, Besucher vom Land, unsittliche Frauen, italienische Straßenorgelspieler, deutsche Blaskapellen, indische Trommler und englische Geigenspieler. Weil der Staat nichts gegen die "tyranny of the lowest mob" unternehme, führt der Mathematiker einen Privatkrieg auf eigene Faust. Lärm ist für Babbage ein Kostenfaktor, der seine potentielle
Arbeitsfähigkeit um $25 \%$ vermindert habe: „I find by some notes, that during about eighty days, I registered one hundred and sixty-five instances, the greater part of which I went out myself to put stop to the nuisance. In several of these cases my whole day's work was destroyed, for they frequently occurred at times when I was giving instruction to my workmen relative to some of the most difficult parts of the Analytical Engine."9

Auch Thomas Carlyle, der schottische Historiker und Schriftsteller, spricht 1853 von Lärm als dem Zerstörer kostbarer Zeit. Er nimmt den Kampf gegen den Lärm auf: Er baut sich eine lärmfreie Oase im Herzen Londons, einen geräuschlosen Raum mit doppelten Wänden, Oberlicht und einem schalldämpfenden Dach. ${ }^{10}$ Bei Carlyle kommt Lärm von außen und kontaminiert die bürgerlichen Innenräume, die Studierzimmer der Gelehrten, die doch gerade als Festung gegen die Außenwelt unter weiblicher Obhut eingerichtet worden sind. Die Briefe und Tagebücher seiner Gattin Jane Welsh Carlyle berichten denn auch von einem Leben, das sich hauptsächlich damit beschäftigt, ihrem Gatten ruhigen Schlaf und lärmfreie Arbeitsgelegenheiten zu verschaffen.

Die historische Annäherung an das Phänomen Lärm, die uns zunächst in die Bibliothek, dann zum Antiphon und schließlich zu Anti-Lärm Pamphleten Mitte des 19. Jahrhunderts geführt hat, zeigt, dass

6 Ebd., 13.

7 Arthur Schopenhauer, Ueber Lerm und Geräusch, in: ders. (Hg.), Parerga und Paralipomena. Kleine philosophische Schriften von Arthur Schopenhauer, Berlin 1988, 551-553 [Berlin 1851], 552.

8 Charles Babbage, Street Nuisances, in: Martin Campbell-Kelly (Hg.), Charles Babbage. Passages from the Life of a Philosopher, New Brunswick 1994, 252-277 [London 1864].

9 Ebd., 265.

10 Theodor Lessing, Der Lärm. Eine Kampfschrift gegen die Geräusche unseres Lebens, Wiesbaden 1908, 24-29. Eine Analyse viktorianischer Arbeitsräume als Rückzugshort der Ruhe unternimmt: John M. Picker, The Soundproof Study: Victorian Professionals, Work Space, and Urban Noise, in: Victorian Studies 42 (1999/2000) 3, 427-453. Über die Schwierigkeiten ein stabiles, kontrollierbares und damit auch lärmfreies Labor für psychologische Experimente einzurichten vgl. Henning Schmidgen, Time and Noise: The Stable Surroundings of Reaction Experiments, 1860-1890, in: Studies in History and Philosophy of Biological and Biomedical Sciences 34 (2003), 259-269. 
Lärm ein Begriff ist, bei dem soziale Konflikte mitschwingen. Die dominierenden Konflikte sind Mitte des 19. Jahrhunderts der Klassen- und der Kulturkampf. Wenn zu diesem Zeitpunkt von Lärm die Rede ist, geht es immer auch um die unbeabsichtigten Nebenfolgen der Moderne (Stadt, Masse, Verkehr, Technik), es geht um die Ängste des Bürgertums vor den Unterschichten, vor Produktivitätsverlusten, vor Dekadenz und davor, von den ,Massen ' in den Städten überrannt zu werden. Im Reden vom Lärm spiegeln sich auch die Dissonanzen zwischen dem bürgerlichen Staat und dem Klerus, zwischen den Anhängern von Säkularisierung und den Vertretern der Kirche. Wenn nun der Versuch unternommen wird Lärm zu historisieren, kann immer nur von einem Wahrnehmungsphänomen die Rede sein. Zwei Punkte möchte ich herausgreifen: Erstens stellt sich die Frage nach der Historisierung des Lärmdiskurses, wobei ich mich auf die Zeit seit etwa 1850 konzentriere, weil Lärm zu diesem Zeitpunkt als negativ konnotierter Schlüsselbegriff des Modernisierungsprozesses besetzt wird ${ }^{11}$. Zweitens geht es um die damit verknüpfte Frage nach der Resonanz von Lärm in der Historiographie bzw. nach dem Zugriff der Geschichte auf den Untersuchungsgegenstand Lärm.

\section{Lärm und Geräusch}

Was ist Lärm? Die etymologischen Ursprünge des deutschen Terminus Lärm bzw. des Verbs lärmen liegen im italienischen
Kriegsruf ,all arme“ bzw. dessen französischen Pendant „al arme“ im 15. Jahrhundert. ${ }^{12} \mathrm{Im}$ 16. Jahrhundert nimmt der Terminus die allgemeinere Bedeutung „Auflauf, Zusammenlauf, Zusammenrottung einer Menge“ an. Seit dem 17. Jahrhundert ist die Bedeutung ,wildes Geschrei, Geräusch, Tosen, Getöse" in den Wörterbüchern verzeichnet. Im 19. Jahrhundert kommt die Bedeutung des Lärmens als „Aufsehen erregen“ dazu. Mitte des 19. Jahrhundert entsteht eine Ausdifferenzierung zwischen den Begriffen Lärm und Geräusch. Der deutsche Physiologe Hermann Helmholtz unternimmt in seiner 1863 erschienen Studie „Die Lehre von den Tonempfindungen" erstmals den Versuch, Physik, Physiologie, Musikwissenschaft und Ästhetik in einer „Theorie der Musik“ $\mathrm{zu}$ vereinen. Helmholtz führt eine Trennung zwischen Klang und Geräusch ein, und bezeichnet erstere als ,periodische Bewegungen der tönenden Körper", letztere als ,nicht periodische Bewegung“. 13 Helmholtz verwendet in seiner Grundlegung der modernen Akustik also nicht den Begriff Lärm, sondern Geräusch um nicht periodische Schwingungen zu bezeichnen. Die in der deutschen Sprache signifikante Unterscheidung zwischen Lärm und Geräusch wird in der englischen Sprache nicht gemacht: Hier bezeichnet noise sowohl Lärm als auch Geräusch. In den 1940er Jahren findet das englische noise schließlich auch Verwendung in der Sprache der Elektronik und Informationstheorie als Bezeichnung von Übertragungsstörungen, die im Deutschen Rauschen genannt wer-

11 Lärm ist in dem auf politische Begriffe konzentrierten Forschungsprojekt der historischen Semantik keine Aufmerksamkeit zuteil geworden. Doch auch dieser Begriff wird im Modernisierungsprozess mit neuen Sinninhalten aufgefüllt und stellt deshalb einen aufschlussreichen Indikator für gesellschaftlichen Wandel dar. Siehe Otto Brunner/Werner Conze/Reinhard Koselleck (Hg.), Geschichtliche Grundbegriffe. Historisches Lexikon zur politisch-sozialen Sprache in Deutschland, Stuttgart 1972. Vgl. die Einleitung von Reinhard Koselleck in Band 1, XIIIXXVII.

12 „Lärmen“, in: Johann Heinrich Zedler (Hg.), Grosses vollständiges Universallexikon aller Wissenschaften und Künste, Halle, Leipzig 1737, 202; „Lärm, Lärmen“, in: Jakob und Wilhelm Grimm (Hg.), Deutsches Wörterbuch, Leipzig 1885, 202-207.

13 Hermann von Helmholtz, Die Lehre von den Tonempfindungen als physiologische Grundlage für die Theorie der Musik, Frankfurt a. M. 1981 [Braunschweig 1863], 16. 
den. ${ }^{14}$ In der englischen Sprache sind drei Bedeutungsebenen (Lärm, Geräusch und Rauschen) im Begriff noise vereint. ${ }^{15}$

Damit ist ein semantisches Feld abgesteckt: Lärm muss als ein sozialer Begriff verstanden werden der auf Phänomene zielt, die vom Menschen als Störung wahrgenommen werden. Die Begriffe Klang und Geräusch werden im 19. Jahrhundert physikalisch aufgeladen. Das Rauschen wird seit den 1930er Jahren von der Elektrotechnik und in den 1950er Jahren von der neu entstehenden Informationstechnik besetzt, wobei die Labors der amerikanischen Telefongesellschaften für die Entwicklung beider Forschungsfelder eine Schlüsselrolle einnehmen.

\section{Kultiviertes Ohr und der Lärm der Kultur}

Welche Quellen vermitteln Lärm? Geräusche sind flüchtig, sie verschwinden mit der Zeit. Die graphische Aufzeichnung schafft Abhilfe: Bereits Helmholtz beschreibt ein Verfahren, das tönende, d.h. „schwingende Körper ihre Bewegungen direct aufs Papier schreiben" lässt, um die Schwingungen dem ,Auge übersichtlicher darzulegen, als das durch weitläufige Beschreibungen geschehen kann. " ${ }^{16}$ Mit Hilfe von Technik ist, seit der Erfindung des Phonographen in den 1870er Jahren und des Magnettonbands in den 1930er Jahren, die Speicherung und Wiedergabe von akus- tischen Zeichen möglich. ${ }^{17}$ Wird Lärm als sozialer Begriff bzw. als soziales Konstrukt ${ }^{18}$ definiert, wofür ich in den folgenden Ausführungen plädieren werde, entsteht Lärm dann, wenn Menschen Geräusche als Störung wahrnehmen und diese beklagen, regulieren, messen, politisch bekämpfen, ästhetisch inszenieren oder technisch unterdrücken. Schriften zum Lärm, die fast ausschließlich Schriften gegen den Lärm sind, verdichten sich kurz nach 1900. ${ }^{19}$ Dabei geht es erstens um Bestandesaufnahmen von Lärmarten nach Verursachern (beispielsweise Verkehrs-, Tier-, Haustier-, Hauswirtschafts-, Musik- und Kirchenglockenlärm), zweitens um Erklärungen $\mathrm{zu}$ tiefer liegenden Ursachen des Lärms und drittens um Vorschläge zu seiner Bekämpfung (auf Ebene von Erziehung, Hygiene, Sozial- und Lebensreformen, Technik und Recht). Bei der Ursachenanalyse zeigen sich Muster, auch wenn die Reichweite der Erklärungsansätze variiert. In einer 1903 erschienenen Schrift des Berliner Stadtbauinspektors G. Pinkenburg sind es die wachsenden Städte und die funktionale Ausdifferenzierung zwischen Wohn- und Arbeitsstätten, die für den Lärm verantwortlich gemacht werden. ${ }^{20}$ Auch das Aufkommen von „Menschenmassen“ lasse den Lärmpegel ansteigen und biete dem Individuum keine Möglichkeiten des Entrinnens mehr. Der Ingenieur ist dennoch optimistisch, dass Straßenlärm gerade mit den Mitteln der Technik und Aufklärung der ,Massen“ durch medizinische Ex-

14 John R. Pierce, Phänomene der Kommunikation. Informationstheorie, Nachrichtenübertragung, Kybernetik, Düsseldorf - Wien 1965 [New York 1961], 173-193.

15 „Noise“, in: Encyclopaedia Britannica, Chicago, 1998, 751.

16 Helmholtz, Die Lehre von den Tonempfindungen, 33-35.

17 Aus medienwissenschaftlicher Perspektive siehe Friedrich Kittler, Grammophon, Film, Typewriter, Berlin 1986, 37-173.

18 Eine scharfsinnige Analyse des Konzeptes der ,sozialen Konstruktion‘ bietet Ian Hacking, Was heisst ,soziale Konstruktion"? Zur Konjunktur einer Kampfvokabel in den Wissenschaften, Frankfurt a. M. 1999.

19 Vgl. z. B. G. Pinkenburg, Der Lärm in den Städten und seine Verhinderung, Handbuch der Hygiene, herausgegeben von Theodor Weyl. Dritter Supplement-Band, Jena 1903; Hermann Beuttenmüller, Der rechtliche Schutz des Gehörs, Karlsruhe 1908; Lessing, Der Lärm.

20 Pinkenburg, Der Lärm in den Städten, 6-10. 
perten in Zukunft reduziert werden könnte. In Theodor Lessings „Kampfschrift gegen die Geräusche des Lebens" aus dem Jahre 1908 ist es die „Kultur“" schlechthin, die für Entstehung und Wahrnehmung des Lärmphänomens verantwortlich ist. ${ }^{21}$ „Kultur“ ist dabei ein Synonym für „Rationalisierung, Logisierung, Ethisierung“. ${ }^{22}$ Mit der Kultivierung des Menschen gehe eine Verfeinerung des Gehörsinns einher und gleichzeitig werde immer mehr Lärm verursacht. ${ }^{23}$ Grammophone transportieren Musik jederzeit an immer entlegenere Orte. Automobile ,stöhnen, ächzen, quietschen, hippen und hupen“" 24 , so dass die Erinnerung an Postkutschen und Postillione romantisch nachklinge. Kirchenglocken hätten in den modernen Städten ihre ursprüngliche Aufgabe eingebüßt. Sie störten bloß noch, weil sie in Privatgefühle und Privatgedanken eindringen. Teppiche, Polster und Betten würden in Individualhaushalten unnötig oft und unkoordiniert geklopft, statt dass auch diese Arbeit arbeitsteilig und in Gemeinschaftshaushalten verrichtet würde, was weniger Lärm verursachen würde.

Lärm ist in der Interpretation der Lärm- kritiker seit der zweiten Hälfte des 19. Jahrhundert ein Phänomen der „Kultur“: Weil Menschen sich vom Natur- zum Kulturwesen entwickelt haben, werden sie immer empfindsamer und weil sie Kulturmenschen geworden sind, produzieren sie immer mehr Lärm. Theodor Lessing, der diese Zusammenhänge am explizitesten ausformuliert hat, ruft zum Kampf gegen den Lärm auf und begründet 1908 den „,Deutschen Lärmschutzverband“. Damit lanciert er eine Form der Lärmbekämpfung, die, nicht wie das „Antiphon“, auf dem individuellen Schutz des Körpers, sondern auf eine Veränderung der gesellschaftlichen Verhältnisse hinzielen. Er setzt auf die Mobilisierung von Massen, die lautstark Lärm schlagen sollen: „,Wer Lärm bekämpfen will, muss Lärm schlagen. Wer in den allgemeinen Geschreie und Getöse gehört will werden, der muss es noch zu überschreien und überlärmen suchen, auch dann, wenn er nichts anderes zu lehren hat, als dass Lärmen und Schreien gemein und unsittlich sei.“"25

Nach 1900 entstehen in Europa und den USA Vereine, die sich dem Kampf gegen Lärm verschreiben: ${ }^{26}$ Erstmals in New

21 Lessing, Der Lärm.

22 Ebd., 17. Vgl. allgemein das Kapitel „Lärm und Kultur“, 14-23.

23 Sigmund Freud hat diese Position zwei Jahrzehnte später in der Formel des „Unbehagens der Kultur" verdichtet, siehe Freud, Das Unbehagen, 197-270.

24 Lessing, Der Lärm, 45.

25 Ebd., 91.

$26 \mathrm{Zu}$ Theodor Lessing und dem „Deutschen Lärmschutzverband“ vgl. Lawrence Baron, Noise and Degeneration: Theodor Lessing's Crusade for Quiet, in: Journal of Contemporary History 17 (1982), 165-178; Matthias Lentz, „Ruhe ist die erste Bürgerpflicht“. Lärm, Grossstadt und Nerven im Spiegel von Theodor Lessings „Antilärmverein“, in: Medizin, Gesellschaft und Geschichte. Jahrbuch des Instituts für Geschichte der Medizin der Robert Bosch Stiftung 13 (1994), 81-105; ders., Eine Philosophie der Tat, eine Tat der Philosophie. Theodor Lessings Kampf gegen den Lärm, in: Zeitschrift für Religions- und Geistesgeschichte 50, 3 (1998), 242-264. Zu Wien siehe Peter Payer, Vom Geräusch zum Lärm. Zur Geschichte des Hörens im 19. und 20. Jahrhundert, in: Wolfram Aichinger/Franz X. Eder/Claudia Leitner (Hg.), Sinne und Erfahrung in der Geschichte, Innsbruck 2003, 173-191; Peter Payer, „Grossstadtwirbel“. Über den Beginn des Lärmzeitalters, Wien 1850-1914, in: Informationen zur modernen Stadtgeschichte 2 (2004), 85-103. Zu Hannover: Richard Birkefeld/Martina Jung, Die Stadt, der Lärm und das Licht. Die Veränderung des öffentlichen Raumes durch Motorisierung und Elektrifizierung, Seelze 1994. Vergleichend zu Europa und den USA Karin Bijsterveld, The Diabolical Symphony of the Mechanical Age. Technology and Symbolism of Sound in European and North American Noise Abatement Campaigns, 1900-40, in: Social Studies of Science 31 (2001) H.1, 37-70; Karin 
York 1906 („Society for the Suppression of Unnecessary Noise“), 1908 in Deutschland initiiert durch Theodor Lessing (,Deutscher Lärmschutzverband") und auch in London (,Street-Noise-Abatement-Committee“). Theodor Lessings Verein vermag die Massen nicht zu mobilisieren: Nur gut 1.000 Mitglieder bekennen sich zum Verein, der bereits 1914 wieder aufgelöst wird. Die schwache Resonanz erklärt sich einerseits mit der Zusammensetzung der Vereinigung, die sich ausschließlich aus Bildungsbürgern rekrutiert, zudem mit deren Fixierung auf die Lärmprofile von Geistesarbeitern, die hauptsächlich auf der Ebene der häuslichen und nachbarschaftlichen Sphäre anzusiedeln sind und beispielsweise die Geräusche der Fabriken nicht mit Lärm in Verbindung bringen. ${ }^{27}$ Bezeichnenderweise ist der Verein in den wachsenden Industriestädten des Kaiserreichs denn auch nicht aktiv. Zudem erweist sich Lessings Konzept der Sensibilisierung der Sinne im Kulturprozess als Bumerang, weil die Exponenten des Antilärmvereins nun mit Nervenschwäche und Neurasthenie in Verbindung gebracht werden und Lärm als subjektive Empfindsamkeit mit geringer gesellschaftlicher Resonanz assoziiert wird. ${ }^{28}$

\section{Verwissenschaftlichung und Verrechtlichung}

Ende der 1920er Jahre und Anfang der 1930er Jahre kommt es zu einer zweiten Welle der Formierungsphase von AntiLärmvereinigungen, die sich nun auf nationaler Ebene organisieren: 1928 in Frankreich, 1933 in Großbritannien, 1934 in Österreich und in Holland. Zudem verdichtet sich ein neues Bedeutungsgewebe von Lärm: Gesundheitsverlust und Ineffizienz sind die neuen Referenzpunkte von Lärm. Es geht nicht mehr um das gefährdete Wohlbefinden einiger sensibler Bildungsbürger, sondern um die Gesundheit und Effizienz aller, um eine, scientific analysis of the noise problem anywhere“. ${ }^{29}$ Den Anfangspunkt markiert dabei eine breit angelegte Erhebung und Klassifizierung von Lärm in New York City, wobei nun Dezibel gemessen, Arbeitseffizienzen berechnet, physiologische Effekte beobachtet, neurologische Prozesse analysiert, Materialien getestet, Menschen befragt und Empfehlungen formuliert werden, kurz: das breitmöglichste Arsenal an wissenschaftlichen Methoden zur Anwendung kommt. ${ }^{30}$ Wichtig ist dabei die Akustik, die sich in den USA in den 1920er Jahren an der

Bijsterveld, „The City Of Din“: Decibel, Noise, and Neighbors in the Nederlands, 1910-1980, in: Osiris 18 (2003), 173-193.

27 In den Berichten von Fabrikinspektoren wird der Maschinenlärm thematisiert und mit Ohrenleiden in Verbindung gebracht, für die Schweiz vgl. Fridolin Schuler/Albrecht E. Burckardt, Untersuchungen über die Gesundheitsverhältnisse der Fabrikbevölkerung in der Schweiz mit besonderer Berücksichtigung des Krankenkassenwesens, Aarau 1889, 176. Aufmerksamkeit wird dem Fabriklärm auch in der Arbeitsmedizin zuteil. Siehe Friedrich Röpke, Die Berufskrankheiten des Ohrs und der oberen Luftwege, Wiesbaden 1902; Robert Jütte, Geschichte der Sinne. Von der Antike bis zum Cyberspace, München 2000, 220-222.

28 Lentz, Eine Philosophie der Tat, eine Tat der Philosophie, 257. Zur Neurasthenie im Kaiserreich vgl. Joachim Radkau, Die wilhelministische Ära als nervöses Zeitalter, oder: Die Nerven als Netz zwischen Tempo- und Körpergeschichte, in: Geschichte und Gesellschaft 20 (1994), 211-241.

29 Edward F. Brown/E. B. Dennis/Jean Henry/G. Edward Pendray (Hg.), City Noise. The Report of the Commission Appointed by Dr. Shirles W. Wynne, Commissioner of Health, to Study Noise in New York City and to Develop Means of Abating it, New York 1930, 3; Bijsterveld, The Diabolical Symphony of the Mechanical Age, 51; N.W. McLachlan, Noise. A Comprehensive Survey from Every Point of View, Oxford - London 1935.

30 Wegweisend für die Verwissenschaftlichung der Lärmwahrnehmung ist das Lärmerhebungsprojekt New Yorks: Brown/Dennis/Henry/Pendray, City Noise. 
Schnittstelle von Physik, Architektur, Medizin und Elektro- und Materialwissenschaft etabliert. ${ }^{31}$ Die Schaffung von schalltechnisch kontrollierten Innenräumen (in Konzert- und Kinosälen, Musikund Kinostudios, Parlamenten und Großraumbüros) steht in einem direkten Zusammenhang mit unkontrollierbaren, als Lärmzone wahrgenommenen Außenräumen in den Großstädten. Obwohl Materialen und Bauverfahren entwickelt werden, die Geräusche absorbieren oder eliminieren, zeigt sich, dass der Technik der Geräuschunterdrückung räumliche Grenzen gesetzt sind. Die Akustik experimentiert denn auch nicht bloß in Labors der expandierenden Telefongesellschaften. Städte wie New York mutieren zu Experimentierfeldern, wobei auf eine Objektivierung der Lärmwahrnehmung gezielt wird. Dabei ergänzen Messapparate („Noise Meter“ und "Audiometer") und Sozialforschung (Befragung der Einwohner über ihre Lärmbelästigung) sich gegenseitig. Die Verwissenschaftlichung des Lärmphänomens kann sich nicht ausschließlich auf Apparate stützen, weil sich die Resultate der Apparate und der Sozialforschung nicht decken. „It can be definitely stated that the level of noise is not the sole factor which determines its annoyance as measured by the number of complaints. " ${ }^{32}$ Die Erklärungslücke, die sich zwischen der seit den 1920er Jahren etablierenden Akustik und den Ergebnissen der Sozialforschung auftut, wird zunehmend durch Physiologie und Experi- mentalpsychologie ergänzt. Lärm wird in diesem Zusammenhang an den Nervenzustand eines Individuums zurückgebunden: „The noise seems to act as a catalytic agent or accessory factor, thereby inducing or accentuating a nervous state." 33

Eng verknüpft mit den Bemühungen um Verwissenschaftlichung des Lärms, sind die Bestrebungen der Verrechtlichung zu sehen. Die Versuche durch Verordnungen und Gesetze das Verhalten von Individuen zu steuern, manifestieren sich in den 1930er Jahren zunächst auf der Ebene des Verkehrs. Der Schweizerische Städteverband widmet sich 1930 an seiner Delegiertenversammlung erstmals der Thematik der „Lärmbekämpfung in den Städten“. ${ }^{34}$ Es ist symptomatisch, dass die beiden Referate von Polizeivorständen stammen: Lärm soll durch Regulation reduziert werden und zwar durch „einwandfreie Verkehrsregelung nach einheitlichen Gesichtspunkten“. .35 Als drängendes Problem wird die Anwendung des Signalapparates identifiziert. Die Verkehrsregulierung läuft auf eine Disziplinierung der Straßenbenützer und die Ersetzung von akustischen Zeichen durch visuelle Zeichen hinaus: Tafeln, Sicherheitslinien, Einbahnstraßen, Rechtsvortritt und Beleuchtung sollen das $\mathrm{Hu}-$ pen reduzieren. Mit den Termini Verwissenschaftlichung, Technisierung (durch schalldämpfende Materialen und Bauweisen), Koordination bzw. Steuerung und Verrechtlichung lassen sich neue Aufmerksamkeiten zu Beginn der 1930er Jahre um-

31 Eine exzellente Wissenschafts- und Architekturgeschichte der Akustik bietet Emily Thompson, The Soundscape of Modernity. Architectural Acoustics and the Culture of Listening in America, 1900-1933, Cambridge/Mass. 2002. Für einen technischen Überblick über die Entwicklung der Akustik Robert T. Beyer, Sounds of Our Times. Two Hundred Years of Acoustics, New Brunswick 1999. Einen Überblick über das entstehende Forschungsfeld Akustik der 1920er Jahre stammt aus der Feder des Research Direktor der Akustikabteilung der Bell Telephone Laboratories: Harvey Fletcher, Speech and Hearing, New York 1929.

32 Brown/Dennis/Henry/Pendray, City Noise, 147.

33 McLachlan, Noise, 134.

34 Protokoll über die Verhandlungen der Delegiertenversammlung des Schweizerischen Städteverbandes, Samstag, den 6. und Sonntag den 7. September 1930, in: Schweizerisches Zentralblatt für Staats- und Gemeindeverwaltung, 23/24 (1930), 44-67.

35 Ebd., 59. 
reißen, die Lärm objektivieren und zugleich als soziales Phänomen definieren. In der Regulierung wird die Lösung für soziale Konflikte gesehen. In den 1950er Jahren verstärkt sich dieser Trend. Beispielhaft für diese Entwicklung ist Karl Oftingers Rechtsstudie (und Streitschrift) „Lärmbekämpfung als Aufgabe des Rechts", die 1956 zeitgleich mit der von ihm lancierten „Schweizerischen Liga gegen den Lärm“ erscheint. ${ }^{36}$ Der Jurist Oftinger interpretiert Lärm als ,eine dem Geist des Rechts und meistens dem Buchstaben des Gesetzes widersprechende Aggression". ${ }^{37}$ Dabei operiert Oftinger mit einer Polarisierung von Technik und Recht, wobei die Technik mit Entfesselung (als Folge von Wissenschaft und Technik) und Recht mit Steuerung (durch Recht, Verwaltung und Planung) assoziiert wird. ${ }^{38}$ Die Verrechtlichung von Lärm geht mit einem Prozess der Politisierung durch eine neue soziale Bewegung einher, der als ,,prä-ökologischer Diskurs" interpretiert worden ist, weil er die um 1970 entstehende Umweltbewegung vorwegnehme. ${ }^{39}$ Damit ist das Bedeutungsspektrum von Lärm an seinem vorläufigen Endpunkt angelangt. Lärm wird nun zum Phänomen der „Umweltzerstörung“ und „Umweltverseuchung“"40 und im Rahmen eines Kodifizierungsprozesses zum Objekt staatlicher Regulierung. Diese Prozesse artikulieren sich in der Schweiz um etwa
1970: Indizien dafür sind beispielsweise die erfolgreiche Volksabstimmung eines Verfassungsartikels für Umweltschutz 1971 oder der Erlass einer Lärmschutzverordnung in der Stadt Zürich (als erste Stadt in der Schweiz) im selben Jahr. ${ }^{41}$

\section{Umweltbelastung und Unordnung}

Seit den 1970er Jahren lässt sich erstmals ein Interesse an der Historisierung des Lärms beobachten - und der Zeitpunkt ist kein Zufall. Mit dem Umweltbegriff öffnet sich in den 1960er Jahren ein neues Raster zur Wahrnehmung von Lärm, das sich auch in der historischen Aufmerksamkeit niederschlägt. Für den kanadischen Komponisten Murray R. Schafer ist der Befund, dass die Umwelt zunehmend mit Lärm kontaminiert sei („noise pollution“) Ausgangpunkt zur empirischen Erforschung der akustischen Umwelt (,,sonic environment") und zur Inventarisierung und Beschreibung moderner Geräuschlandschaften im Rahmen des Ende der 1960er Jahre begonnenen kollaborativen internationalen „World Soundscape Projects“.42 Aus diesen Studien entsteht 1977 unter dem Titel „The Tuning of the World“ eine Synthese, die zugleich ein Manifest für eine zukünftige Geräuschforschung darstellt, wobei die Grenzen zwischen Musikpraxis, Umwelt-

36 Karl Oftinger, Lärmbekämpfung als Aufgabe des Rechts, Zürich 1956. Zur historischen Analyse der „Schweizerischen Liga gegen den Lärm“ als soziale Bewegung vgl. Mischa Gallati, Die Schweizerische Liga gegen den Lärm 1956-1966, Zürich 2004.

37 Oftinger, Lärmbekämpfung als Aufgabe des Rechts, IV.

38 Vgl. dazu Karl Oftinger, Konfrontation der Technik mit dem Recht, in: Hans Freyer/Johannes Chr. Papalekas/Georg Weippert (Hg.), Technik im technischen Zeitalter. Stellungsnahmen zur geschichtlichen Situation, Düsseldorf 1965, 247-270.

39 Gallati, Die Schweizerische Liga, 11.

40 Dieter Hentrup, Die Umweltverseuchung Nummer eins. Lärm ist zur ernsthaften Gesundheitsgefahr für Millionen von Menschen geworden, in: Frankfurter Rundschau, 30. September 1978.

41 „Zürich erlässt eine Lärmschutzverordnung“, in: Volksrecht, Nr. 119, 25. Mai 1971.

42 R. Murray Schafer, The Book of Noise, Vancouver 1970; World Soundscape Project/R. Murray Schafer, A Survey of Community Noise By-Laws in Canada, [Burnaby B. C.] 1972; $R$. Murray Schafer, Five Village Soundscapes, The Music of the Environment Series 4, Vancouver/ B. C. 1977; Richard Murray Schafer, The Soundscape. Our Sonic Environment and the Tuning of the World, Rochester, Vermont 1994 [zuvor: The Tuning of the World, New York 1977]. 
aktivismus und Feldforschung unterlaufen werden. Das Buch ist aus Sicht der Geschichtswissenschaft gerade auch deshalb interessant, weil der Vergangenheit ein zentraler Stellenwert für die Interpretation der Gegenwart zukommt. Schafers Ausführungen liegt eine These über den historischen Wandel der Geräuschlandschaften zugrunde: Sie hätten sich von High Fidelity (HiFi = hohe Klangtreue) zu Low Fidelity (LoFi =geringe Klangtreue) gewandelt, wobei die ländliche Gesellschaft (bzw. die alten Zeiten) durch $H i F i$ und die Städte (bzw. die neuen Zeiten) durch $\mathrm{LoFi}$ gekennzeichnet seien. ${ }^{43}$ Der entscheidende Übergang zwischen den beiden Epochen markiert die industrielle Revolution, welche im Laufe der historischen Entwicklung um die elektrische Revolution erweitert wird. Schafers Periodisierung basiert auf einer Kontrastierung von Natur und Technik: „The sounds of nature are being lost under the combined jamming of industrial and domestic machinery“. ${ }^{44}$ Zudem erzählt er eine Verlustgeschichte: Alte Geräusch werden in der Erinnerung im Vergleich zu neuen Geräuschen positiv bewertet: „By comparison with the sounds of modern transportation, those of the trains were rich and characteristic." 45 Schließlich vertritt er die These des gesellschaftlich sanktionierten (seiner Ansicht nach „heiligen“) Lärms, der sich im Modernisierungsprozess von der Religion zur Technik verlagert habe: Während früher der Ritus und der Kult ein Ort des erlaubten Lärms gewesen sei, komme diese Ehre heute den Maschinen zu. Dieser Gedanke ist aus technikhistorischer Perspektive durchaus weitere Überlegungen wert, ein besonders prägnantes Beispiel sind bei- spielsweise die Raketenstarts der Raumschifffahrt.

Der Umweltdiskurs kann als Anlass und strukturierendes Element des Zugriffs der Geschichte auf das Phänomen Lärm bezeichnet werden. Dabei haben besonders die Anti-Lärmvereinigungen das Interesse der Historie auf sich gezogen. Inzwischen gehören die Bürgerinitiativen gegen Lärm, die sich seit der Jahrhundertwende in den Städten der USA und Europa formiert haben, zu den gut erforschten Aspekten von Lärm. ${ }^{46}$

Dabei scheint der umweltgeschichtliche Zugriff auf historische Lärmphänomene den Blick dieser hervorragenden Studien bisweilen etwas zu verstellen. Während Lärm bei Theodor Lessing ein Effekt der „Kultur“ darstellt, sprechen Historiker von „handfesten Umweltproblemen“47 oder „städtischen Umweltbelastungen“48 um die Jahrhundertwende. Dieses Vorgehen wird von Micha Gallati in seiner exzellenten Studie zur „Schweizerischen Liga gegen den Lärm" in den 1950er Jahren selbstkritisch reflektiert: Wenn er diese Vereinigung in einem prä-ökologischen Diskurs verortet, spricht er von einem ,noch nicht Vorhandensein, das immer auf die Leerstelle der Zukunft verweist, die inzwischen wieder zur Vergangenheit geworden ist. (...) Wird eine Vor-Geschichte erzählt, tritt besonders deutlich zu Tage, was Geschichtsschreibung immer ist: Eine nachträgliche Konstruktion der Vergangenheit." 49

Jenseits des Umweltdiskurses gibt es bei Murray R. Schafer in den 1970er Jahren noch andere Thematisierungen von Lärm. ${ }^{50}$ Trotz seiner teilweise etwas der

43 Schafer, The Soundscape, 43

44 Ebd., 84.

45 Ebd., 81.

46 Wie weiter oben in Fußnote 26 angedeutet.

47 Lentz, „Ruhe ist die erste Bürgerpflicht“, 103.

48 Payer, Vom Geräusch zum Lärm, 178.

49 Gallati, Die Schweizerische Liga, 11.

50 Schafer, The Soundscape. 
Nostalgie verpflichteten Position, wird Lärm bei ihm als kulturell codiertes Wahrnehmungsphänomen verstanden. Beachtlich scheint mir, dass er die gesellschaftliche und musikalische Entwicklung der letzten hundert Jahre konsequent aufeinander bezieht. Dies führt zu interessanten Beobachtungen: Etwa, dass die Lautsphären von Gesellschaften in den Konzertsälen verdoppelt werden. Im 18. Jahrhundert sind es die Laute der Natur, die in der Musik wiedergegeben werden, während im industriellen Zeitalter des 19. Jahrhunderts die Bässe aller Saiteninstrumente verstärkt und das Cembalo durch das lautere Klavier ersetzt werden. Ludwig van Beethovens geballte Sforzandi bereiten den Weg zu den massiv vergrößerten Orchestern von Richard Wagner und Hector Berlioz. Im 20. Jahrhundert wird Lärm gar zum Programm von Musik erhoben, erstmals 1913 bei Luigi Russolos futuristischem Manifest „L'arte dei Rumori“, eine Position die von John Cage in den 1950er Jahren wieder aufgenommen wird. Damit ist die seit Hermann von Helmholtz in der klassischen Musik konstitutive Unterscheidung von Ton und Geräusch in Frage gestellt und zugleich wird die Codierung von Lärm als störendes Geräusch subversiv gewendet. ${ }^{51}$ Die Engführung von Musik- und Gesellschaftsentwicklung verweist auf Gegenläufigkeiten: Während in den 1960er Jahren im Arbeitsschutz Grenzwerte für Lärm eingeführt werden, lassen Rockbands den Lärmpegel ausschlagen. Auch methodisch ist der Ansatz Schafers interessant: Zur Er- forschung vergangener Klangwelten empfiehlt er neben der Analyse literarischer Berichte auch morphologische Studien: Beispielsweise eine Analyse der verwendeten Materialien, Transportmittel und Kommunikationssignale einer Gesellschaft als Indiz für epochenspezifische Klänge, wobei besonders die Übergänge aufschlussreich seien. Schließlich empfiehlt er eine Analyse von Lärmverordnungen, nicht weil sie als Seismograph für realen Lärm dienen könnten, sondern weil damit die symbolische Dimension des Lärms und die akustischen Phobien einer Gesellschaft sichtbar würden. Schafers Beschreibung von Klangwelten zielen auf die Forderung nach der Aufwertung des Hörsinns und eine Schulung der akustischen Kompetenz durch „Hörschärfung“. Diesem Zweck soll die Schaffung eines Archivs von aussterbenden Klangfarben dienen. ${ }^{52}$ Beispiele für potentielle Archivtöne wären das Klingeln alter Registrierkassen, das Stampfen von Butter im Fass oder das Klicken alter Kameras. Auch die Übertragung von Naturgeräuschen mittels ,environmental radios" in die Städte propagiert er zur Hörschärfung. Es ist also keineswegs ein technikfreies „Zurück zur Natur“, das hier vom Umweltaktivisten gefordert wird. Ganz im Gegenteil: Durch Speicher- und Übertragungstechniken soll sie ,zurück sprechen" ${ }^{\text {"53 }}$. Dennoch erzählt Schafer eine Geschichte, die davon handelt, dass die Geräusche früher klangvoller tönten und dass Lärm weniger störend war. Damit geht er der Antilärmliteratur auf den Leim, die im-

51 Ebd., 5. Zur Technisierung der Musik vgl. Hans-Joachim Braun, Technik im Spiegel der Musik des frühen 20. Jahrhundert, in: Technikgeschichte 59, 2 (1992), 109-131; ders., „I sing the body electric“. Der Einfluss der Elektroakustik und Elektronik auf das Musikschaffen im 20. Jahrhundert, in: Technikgeschichte 61 (1994) H. 4, 353-373; Trevor J. Pinch/Karin Bijsterveld, „Should One Applaud?“. Breaches and Boundaries in the Reception of New Technology in Music, in: Technology and Culture 44 (2003), 536-559. Zur Lärmdebatte in der Musik des 20. Jahrhunderts siehe Thompson, The Soundscape of Modernity, 130-144.

52 Im Rahmen des „World Soundscape Project“ etablieren sich die Field Records, die inzwischen zur Errichtung von groß angelegten akustischen Archiven geführt haben: Vgl. http:// www.sfu.ca/sonic-studio/srs/ (19.12.2005).

53 Schafer, The Soundscape, 236. 
mer auch davon handeln, dass es früher besser war.

Alain Corbin hat eine der seltenen historischen Studien vorgelegt, die sich mit der Bedeutung von Klängen für die soziale Ordnung beschäftigen. ${ }^{54}$ „Die Sprache der Glocken" handelt vom Kirchengeläut als akustisches Kommunikationssystem im Frankreich des 19. Jahrhunderts. Auch Corbin kontrastiert ein ländliches und ein städtisches Wahrnehmungssystem: Die Kritik an Glockenlärm sei eine städtische Tradition und artikuliere sich bereits im 16. Jahrhundert. ${ }^{55} \mathrm{Im}$ ländlichen Raum hingegen gebe es nur wenig Intoleranz gegenüber dem Glockengeläut: Etwa gegenüber dem Läuten der Totenglocken in Zeiten von Epidemien, das untersagt wird, aus Angst durch akustische Signale die Epidemie zu reproduzieren und Angst und Schrecken zu verbreiten. Ebenfalls als störendes Geräusch wahrgenommen werde im 19. Jahrhundert das abendliche Läuten im Sommer um zehn Uhr, weil damit die früh aufstehenden Arbeiter um ihren Schlaf gebracht werden. In der Stadt hingegen wird morgendliches Läuten als lästig empfunden, weil sie als Eingriff in die individuelle Freiheit wahrgenommen werden. Die Schmähungen der Glocke durch die Städter nehmen im Laufe des 19. Jahrhunderts generell gewaltig zu, mit den 1860er Jahren als Wendepunkt. Schauplatz der Polemik gegen Lärm sind Thermalkurorte, wo Städter auf Landbewohner treffen und ein „Recht auf Ruhe“ fordern. Hinter dem Glockenstreit stehen ein Wandel im Ein- schätzungssystem, eine Infragestellung von traditionellen Klangordnungen und ein Wiederaufflammen der Kulturkämpfe zwischen der Kirche und den Anti-Klerikern. Die alten Klangbotschaften, welche während vielen Jahrhunderten das Leben der Landbewohner strukturiert hatten, werden nicht mehr verstanden und mutieren zum Lärm.

Peter Bailey hat in einem 1996 erschienen Essay darauf gedrängt, den symbolischen Dimensionen von Lärm mehr Bedeutung zuzumessen, als dies bislang in der Geschichtsschreibung der Fall war. ${ }^{56}$ Dabei sei die Symbolik vor dem Hintergrund sozialer Ordnungen zu diskutieren, ein Vorgehen, das er der amerikanischen Kulturanthropologin Mary Douglas entlehnt. In ihrer Studie „Reinheit und Gefährdung“ definiert sie Schmutz als Unordnung, bzw. als Verstoß gegen die soziale Ordnung. ${ }^{57}$ Bailey überträgt diese Definition von Schmutz auf Lärm. Derart gewendet ist Lärm ein Verstoß gegen die Ordnung, d.h. Unordnung und Störung. Diese Ausweitung des Blicks auf Lärm öffnet meiner Ansicht nach neue Interpretationsansätze für Prozesse, die bislang vielleicht etwas einseitig aus der Perspektive der Umweltgeschichtsschreibung interpretiert worden sind. Ein Beispiel dafür ist Karl Oftingers Abhandlung „Lärm als Aufgabe des Rechts“. Wenn Oftinger Lärm als „eine dem Geist des Rechts und meistens dem Buchstaben des Gesetzes widersprechende Aggression" 58 bezeichnet, zielt er neben Technikkritik (die nicht gleichzusetzen ist

54 Alain Corbin, Die Sprache der Glocken. Ländliche Gefühlskultur und symbolische Ordnung im Frankreich des 19. Jahrhunderts, Frankfurt a. M. 1995 [Paris 1994].

55 Ebd., zum Verlust des Sinns für Klangbotschaften seit den 1860er Jahren vgl. speziell 402-415. Zu den Geräuschen der europäischen Städte der frühen Neuzeit vgl. David Garrioch, Sounds of the City: The Soundscape of Early Modern European Towns, in: Urban History 30 (2003) H. 1, 5-25. Ebenfalls für die frühe Neuzeit: Jean-Pierre Gutton, Bruits et sons dans notre histoire. Essai sur la reconstitution du paysage sonore, Paris 2000.

56 Peter Bailey, Breaking the Sound Barier. A Historian Listens to Noise, in: Body \& Society 2 (1996) H. 2, 49-66.

57 Mary Douglas, Reinheit und Gefährdung. Eine Studie zur Vorstellung von Verunreinigung und Tabu, Berlin 1985 [London 1966], 12.

58 Oftinger, Lärmbekämpfung als Aufgabe des Rechts, IV. 
mit Umweltbewusstsein) durchaus auf eine Gefährdung der Ordnung durch Technik, die sich seiner Ansicht nach am dramatischsten am Phänomen Lärm manifestiert: „Das Recht ist von der Technik überspielt, ja stellenweise ausmanövriert worden. Das Ergebnis ist die immer weiter schreitende Kapitulation der Rechtsordnung vor den Ansprüchen der Technik. "59 Er spricht von „Degenerationserscheinungen“ des Rechts und einer „Rebarbarisierung“ der Gesellschaft. In diesen Äußerungen lassen sich durchaus Parallelen zum Antilärmdiskurs Mitte des 19. Jahrhunderts ausmachen, der auch von Degenerationsängsten gekennzeichnet war. Die niederländische Technikhistorikerin Karin Bijsterveld entwickelt den weiter zu verfolgenden Gedanken, dass „Lärmobsessionen“ in einer Korrelation zu sozialem Wandel stünden, und die Thematisierung von Lärm in Perioden schneller Modernisierung zunehme. ${ }^{60}$ Diese These scheint mir verfolgenswert, weil Lärmobsessionen nicht einfach als Reaktion auf die Zunahme von Geräuschen interpretiert werden können, sondern als Produkt kommunikativer Prozesse analysiert werden müssen, die im Zusammenhang mit sozialem Wandel stehen. Peter Bailey hat denn auch angeregt, Lärm auch als kommunikative Ressource anzuschauen, welche die Fähigkeit habe ,to appropriate, reconfigure or transgress boundaries."61 Derart gefasst, öffnet sich ein Forschungsfeld, das sich mit dem Einsatz von Lärm als soziale Waffe und Mittel der Macht beschäftigen könnte. ${ }^{62}$

Die Wahrnehmung und Thematisierung von Lärm in sozialen Interaktionen in Mikrokontexten gehört ebenfalls zu den bislang eher vernachlässigten Themen. Karin Bijsterveld hat vorgeführt, wie ein solcher Zugriff funktionieren könnte, indem sie Nachbarschaftskonflikte um Lärm in den Niederlanden in den 1930er Jahren analysiert, als Musikapparate (wie Grammophone und Radios) zum Massenkonsumgut avancieren und die Polizei erstmals akustische Messgeräte verwendet. ${ }^{63}$ Aufschlussreich könnte auch der Blick auf die Geschlechterperspektive von Lärmkonflikten und Lärmbekämpfungsmaßnahmen sein: Ich verweise auf Phänomene wie die weibliche Konnotation der zu lärmfreien Zonen deklarierten Innenräume in der bürgerlichen Gesellschaft ${ }^{64}$ oder die allgemeine Frage, wie Lärm und Ruhe in sozialen Interaktionen konnotiert sind, beispielsweise in psychiatrischen Kliniken in der ersten Hälfte des 20. Jahrhunderts, wo speziell Frauen als laut und störend wahrgenommen werden. ${ }^{65}$ Auch die experimentelle Erforschung des Lärms (bspw. in der Akustik und Physiologie) wäre ein Feld für weitere Forschungen, wobei Emily Thompson und Karin Bijsterveld das Terrain mit hervorragenden Studien bereits geebnet ha-

59 Ebd.

60 Bijsterveld, The Diabolical Symphony, 43.

61 Bailey, Breaking the Sound Barier, 6.

62 Zur Hundejagd als Lärmquelle im adeligen Hofleben des 14.-16. Jahrhundert vgl. Simon Teuscher, Hunde am Fürstenhof. Köter und „edle wind“ als Medien sozialer Beziehungen vom 14. bis 16. Jahrhundert, in: Historische Anthropologie 6 (1998), 364-368. Dem Lärm durch die bellenden Hunde kommt in der höfischen Gesellschaft die Funktion einer akustischen Raumdurchdringung und eines Mediums der Kohäsion zu.

63 Bijsterveld, „The City Of Din“, 185-193. Auch Nutzungskonflikte, die durch technische Apparate wie Radios oder Medizinalgeräte ausgelöst werden, könnten ein Feld für technikhistorische Forschungen sein. Vgl. Ludwig Ebermayer, Der Arzt im Recht. Rechtliches Handbuch für Ärzte, Leipzig 1930, 281-283.

64 Ansätze dazu verfolgt Picker, The Soundproof Study, 427-453.

65 Marietta Meier, Eingriffe ins Gehirn ,schwieriger“ Patientinnen und Patienten. Psychochirurgie im Kanton Graubünden, in: Ursula Jecklin/Silke Redolfi/Silvia Hofmann (Hg.), frauenKörper: Beiträge zur Frauen- und Geschlechtergeschichte Graubündens im 19. und 20. Jahrhundert, Zürich 2005, 252-255. 
ben. ${ }^{66}$ Eine kulturwissenschaftliche $\mathrm{He}$ rangehensweise würde auch naturwissenschaftliche Beschreibungen von Lärm als kulturell codiertes und sozial geprägtes Phänomen analysieren. Dabei könnte auch ein Blick auf die biochemische Antwort der Pharmaindustrie auf das Lärmproblem von Bedeutung sein. Ein Hinweis muss genügen: Das Basler Pharmaunternehmen Geigy publiziert 1960 eine Broschüre zu den physiologischen Auswirkungen des Lärms und wirbt dabei mit dem Slogan „Vom Lärm des Tages zur Ruhe der Nacht“" prominent für das Schlaf- und Beruhigungsmittels „Medomin“. 67 Was das mechanische „Antiphon“ im 19. Jahrhundert, ist das synthetische Barbiturat Mitte des 20. Jahrhunderts: eine Lärmbekämpfungstechnik für den individuellen Gebrauch.

In der Auslegeordnung der longue durée zeigt sich, dass Lärm ein Resonanzphänomen darstellt, das kulturalistisch betrachtet und gewendet werden kann. Es ist aufschlußreich, darauf zu achten, was auch noch mitschwingt, wenn Lärm erlitten, bekämpft, verrechtlicht oder verwissenschaftlicht wird. Auch eine Analyse des Gegenbegriffs von Lärm, nämlich Ruhe bzw. Stille, könnte produktiv für eine kulturwissenschaftliche Analyse von Lärm sein, wie mein abschließendes Beispiel zeigt.

\section{Stille und Rauschen: Ausklang}

Ausgerüstet mit Mikrophon und Magnettonband streift Hans Traber 1950 durch die Schweizer Wälder. ${ }^{68}$ Traber, der im Laufe der folgenden Jahre in der Schweiz zum berühmten Fernsehnaturforscher aufsteigen wird, sammelt Vogelstimmen mit seinem Tonband. Auf Vinyl gepresst er- obert die mediatisierte Wahrnehmung der Naturgeräusche in den folgenden Jahrzehnten die Grammophone und Stereoanlagen. Die Geräusche der Natur werden zur Musik (nicht zufällig werben die Tonträger mit „Meistersingern“), die Natur wird in der isolierten Umgebung der Stube jederzeit akustisch wahrnehmbar, die Stimmen der einzelnen Vögel sind isoliert zu hören. Was der Hörer nicht weiß, sind die Schwierigkeiten, die der Aufnahme vorangegangen sind: ${ }^{69}$ Die siebzig Kilogramm schwere Apparatur muss in den Wald geschleppt werden, bereits um vier Uhr morgens wird die Apparatur in Stellung gebracht, dann wenn die Vögel sich akustisch bemerkbar machen. Schließlich sind es nicht bloß die erhofften Vogelstimmen, die auf dem Tonband festgehalten werden, sondern störende Geräusche, Lärm: „Man darf nicht vergessen, dass die Empfindlichkeit des Aufnahmegeräts so hoch ist, dass man unsere liebe SBB [Schweizerische Bundesbahnen] bereits aus zwei bis drei Kilometer Entfernung im Kontrolllautsprecher vernimmt. Es ist somit gerade der Frühzug, der uns den ersten Streich spielt! Dann kommen die erwachsenen Hähne in den nahen Bauerngehöften, vielleicht auch noch ein eifriger Hofhund, der erste Sonntag-, Spaziergänger' mit seinem knatternden Motorrad, die Turmuhr, die weit vernehmlich die Zeit verkündet, ein Pferdekarren, der zum Grasen fährt, Mähmaschinen und Glocken vom weidenden Vieh. Ist dann der Tag vollends erwacht, gibt es in unserem Mittelland nur wenige Gebiete, in die der Lärm der Zivilisation nicht eindringt. Hat man nach langem Suchen einen solchen Platz gefunden, so kann es dann doch passieren, dass einem die Ätherwellen die Mikrophonzuleitung bearbeiten und die ganze

66 Bijsterveld, The Diabolical Symphony; Bijsterveld, „The City Of Din“; Thompson, The Soundscape of Modernity.

67 K.D. Kryter/E. Grandjean, Die Wirkungen des Lärms auf den Menschen, Basel 1960.

68 Hans A. Traber, So singen unsere Vögel, Zürich 1973. [Eine frühere Ausgabe dieses Tonträgers erschien bei Oldenbourg 1959].

69 Hans Traber, Der gefangene Gesang, in: Du. Die Zeitschrift für Kultur 4 (1950), 33 
Sendung von Radio Beromünster inmitten der Vogelstimmen umhergeistert. " Das Aufnahmegerät bringt zutage, was der Wahrnehmungsapparat des Menschen auszublenden gelernt hat, er kann weghören, und Geräusche, die er nicht hören will, bis zu einem gewissen Grad ausfiltern. Zudem produziert Technik neuen Lärm, welcher der Aufmerksamkeit der Menschen entgeht, weil sie weniger empfindlich sind als das Mikrophon und das Tonband. Die elektroakustischen Interferenzen des Radios stören die Übertragung der Vogelstimmen, es entsteht ein unverständliches Rauschen. Die Stille der Natur, welche im natur- und heimatschützerischen Ambiente der 1950er Jahre als Antipode zum Lärm der Kultur inszeniert wird, entlarvt sich spätestens durch ihre Mediatisierung als Unmöglichkeit. Stille als Illusion: Diese Position wird, wohl kaum zufällig, fast gleichzeitig auch in der Avantgardemusik auf den Punkt gebracht. John Cage lässt 1952 seine Komposition „4'33"“ a aufführen. Der Pianist spielt nichts. Doch statt Stille vernehmen die Zuhörer ihre eigenen Geräusche.

70 Ebd. 